PROCEEDINGS OF THE

AMERICAN MATHEMATICAL SOCIETY

Volume 137, Number 3, March 2009, Pages 899-910

S 0002-9939(08)09585-3

Article electronically published on September 24, 2008

\title{
MINIMALITY OF THE BOUNDARY OF A RIGHT-ANGLED COXETER SYSTEM
}

\author{
TETSUYA HOSAKA
}

(Communicated by Alexander N. Dranishnikov)

\begin{abstract}
In this paper, we show that the boundary $\partial \Sigma(W, S)$ of a rightangled Coxeter system $(W, S)$ is minimal if and only if $W_{\tilde{S}}$ is irreducible, where $W_{\tilde{S}}$ is the minimum parabolic subgroup of finite index in $W$. We also provide several applications and remarks. In particular, we show that for a right-angled Coxeter system $(W, S)$, the set $\left\{w^{\infty} \mid w \in W, o(w)=\infty\right\}$ is dense in the boundary $\partial \Sigma(W, S)$.
\end{abstract}

\section{INTRODUCTION AND PRELIMINARIES}

The purpose of this paper is to study dense subsets of the boundary of a Coxeter system. A Coxeter group is a group $W$ having a presentation

$$
\left.\langle S|(s t)^{m(s, t)}=1 \text { for } s, t \in S\right\rangle,
$$

where $S$ is a finite set and $m: S \times S \rightarrow \mathbb{N} \cup\{\infty\}$ is a function satisfying the following conditions:

(1) $m(s, t)=m(t, s)$ for each $s, t \in S$,

(2) $m(s, s)=1$ for each $s \in S$, and

(3) $m(s, t) \geq 2$ for each $s, t \in S$ with $s \neq t$.

The pair $(W, S)$ is called a Coxeter system. If, in addition,

(4) $m(s, t)=2$ or $\infty$ for each $s, t \in S$ with $s \neq t$,

then $(W, S)$ is said to be right-angled. Let $(W, S)$ be a Coxeter system. Then $W$ has the word metric $d_{\ell}$ defined by $d_{\ell}\left(w, w^{\prime}\right)=\ell\left(w^{-1} w^{\prime}\right)$ for each $w, w^{\prime} \in W$, where $\ell(w)$ is the word length of $w$ with respect to $S$. For a subset $T \subset S, W_{T}$ is defined as the subgroup of $W$ generated by $T$, and is called a parabolic subgroup. If $T$ is the empty set, then $W_{T}$ is the trivial group. A subset $T \subset S$ is called a spherical subset of $S$ if the parabolic subgroup $W_{T}$ is finite.

Every Coxeter system $(W, S)$ determines a Davis complex $\Sigma(W, S)$ which is a CAT(0) geodesic space $(6,7], 8,20])$. Here the 1-skeleton of $\Sigma(W, S)$ is the Cayley graph of $W$ with respect to $S$. The natural action of $W$ on $\Sigma(W, S)$ is proper, cocompact and by isometries. If $W$ is infinite, then $\Sigma(W, S)$ is noncompact and $\Sigma(W, S)$ can be compactified by adding its ideal boundary $\partial \Sigma(W, S)([4,[7, \S 4])$.

Received by the editors November 20, 2006, and, in revised form, March 25, 2008.

2000 Mathematics Subject Classification. Primary 20F65, 20F55.

Key words and phrases. Boundaries of Coxeter groups.

The author was partially supported by the Grant-in-Aid for Young Scientists (B), The Ministry of Education, Culture, Sports, Science and Technology, Japan (No. 18740025).

(C)2008 American Mathematical Society Reverts to public domain 28 years from publication 
This boundary $\partial \Sigma(W, S)$ is called the boundary of $(W, S)$. We note that the natural action of $W$ on $\Sigma(W, S)$ induces an action of $W$ on $\partial \Sigma(W, S)$ by homeomorphisms.

A subset $A$ of a space $X$ is said to be dense in $X$ if $\bar{A}=X$. A subset $A$ of a metric space $X$ is said to be quasi-dense if there exists $N>0$ such that each point of $X$ is $N$-close to some point of $A$. Suppose that a group $G$ acts on a compact metric space $X$ by homeomorphisms. Then $X$ is said to be minimal if every orbit $G x$ is dense in $X$.

For a negatively curved group $\Gamma$ and the boundary $\partial \Gamma$ of $\Gamma$, it is known that each orbit $\Gamma \alpha$ is dense in $\partial \Gamma$ for any $\alpha \in \partial \Gamma$, that is, $\partial \Gamma$ is minimal (9]). We note that Coxeter groups are nonpositive curved groups and not negatively curved groups in general. Indeed, there exist examples of Coxeter systems whose boundaris are not minimal (cf. [14, 16]). The purpose of this paper is to investigate when the boundary of a Coxeter system is minimal.

In [14, Theorem 1], we have obtained a sufficient condition of a Coxeter system $(W, S)$ such that some orbit of the Coxeter group $W$ is dense in the boundary $\partial \Sigma(W, S)$. After some preliminaries in Section 2, we first show that the boundary of such a Coxeter system is minimal; that is, we prove the following theorem in Section 3 .

Theorem 1. Let $(W, S)$ be a Coxeter system. Suppose that $W^{\left\{s_{0}\right\}}$ is quasi-dense in $W$ with respect to the word metric and $o\left(s_{0} t_{0}\right)=\infty$ for some $s_{0}, t_{0} \in S$, where $o\left(s_{0} t_{0}\right)$ is the order of $s_{0} t_{0}$ in $W$. Then

(1) $\partial \Sigma(W, S)$ is minimal, and

(2) $\left\{w^{\infty} \mid w \in W, o(w)=\infty\right\}$ is dense in $\partial \Sigma(W, S)$.

Here $W^{\left\{s_{0}\right\}}=\left\{w \in W \mid \ell(w t)>\ell(w)\right.$ for each $\left.t \in S \backslash\left\{s_{0}\right\}\right\} \backslash\{1\}$ and $w^{\infty}$ is the point of $\partial \Sigma(W, S)$ to which the sequence $\left\{w^{i} \mid i \in \mathbb{N}\right\} \subset \Sigma(W, S)$ converges in $\Sigma(W, S) \cup \partial \Sigma(W, S)$.

In Sections 4 and 5, we investigate right-angled Coxeter groups and we prove the following main theorem.

Theorem 2. For a right-angled Coxeter system $(W, S)$, the boundary $\partial \Sigma(W, S)$ is minimal if and only if $W_{\tilde{S}}$ is irreducible.

Here for $T \subset S, W_{T}$ is said to be irreducible if $W_{T}$ does not split as a product $W_{T_{1}} \times W_{T_{2}}$ for any nonempty subsets $T_{1}$ and $T_{2}$ of $T$, and $W_{\tilde{S}}$ is the minimum parabolic subgroup of finite index in $(W, S)$, that is, for the irreducible decomposition $W=W_{S_{1}} \times \cdots \times W_{S_{n}}, \tilde{S}=\bigcup\left\{S_{i} \mid W_{S_{i}}\right.$ is infinite $\}$ (11]).

We provide several applications of Theorem 2 in Sections 5 and 6 . In particular, we show the following corollary.

Corollary 3. For a right-angled Coxeter system $(W, S)$, the set $\left\{w^{\infty} \mid w \in W, o(w)\right.$ $=\infty\}$ is dense in the boundary $\partial \Sigma(W, S)$.

In Section 6, we give some remarks on dense subsets of boundaries of $\operatorname{CAT}(0)$ groups.

\section{Lemmas on CoXeter groups}

In this section, we show some lemmas for (right-angled) Coxeter groups which are used later.

We first give some definitions. 
Definition 2.1. Let $(W, S)$ be a Coxeter system and $w \in W$. A representation $w=s_{1} \cdots s_{l}\left(s_{i} \in S\right)$ is said to be reduced, if $\ell(w)=l$, where $\ell(w)$ is the minimum length of a word in $S$ which represents $w$.

Definition 2.2. Let $(W, S)$ be a Coxeter system. For each $w \in W$, we define $S(w)=\{s \in S \mid \ell(w s)<\ell(w)\}$. For a subset $T \subset S$, we also define $W^{T}=\{w \in$ $W \mid S(w)=T\}$.

The following lemma is known.

Lemma 2.3 ([1, 5, p.37], [18]). Let $(W, S)$ be a Coxeter system.

(1) Let $w \in W$ and let $w=s_{1} \cdots s_{l}$ be a representation. If $\ell(w)<l$, then $w=s_{1} \cdots \hat{s}_{i} \cdots \hat{s}_{j} \cdots s_{l}$ for some $1 \leq i<j \leq l$.

(2) For each $w \in W$ and $s \in S, \ell(w s)$ equals either $\ell(w)+1$ or $\ell(w)-1$, and $\ell(s w)$ also equals either $\ell(w)+1$ or $\ell(w)-1$.

(3) For each $w \in W, S(w)$ is a spherical subset of $S$; i.e., $W_{S(w)}$ is finite.

We can obtain the following lemma from Lemma 2.3 (3).

Lemma 2.4. Let $(W, S)$ be a Coxeter system and let $T$ be a maximal spherical subset of $S$. Then $W^{T}$ is quasi-dense in $W$.

Proof. Let $w \in W$. There exists an element $w^{\prime}$ of longest length in the coset $w W_{T}$. Then we show that $S\left(w^{\prime}\right)=T$.

Let $t \in T$. Since $w^{\prime} t \in w^{\prime} W_{T}=w W_{T}$ and $w^{\prime}$ is the element of longest length in $w W_{T}, \ell\left(w^{\prime} t\right)<\ell\left(w^{\prime}\right)$, i.e., $t \in S\left(w^{\prime}\right)$. Thus $T \subset S\left(w^{\prime}\right)$. Now $T$ is a maximal spherical subset of $S$ and $S\left(w^{\prime}\right)$ is a spherical subset of $S$ by Lemma 2.3 (3). Hence $S\left(w^{\prime}\right)=T$ and $w^{\prime} \in W^{T}$.

Here $d_{\ell}\left(w, w^{\prime}\right) \leq \max \left\{\ell(v) \mid v \in W_{T}\right\}$. Hence $W^{T}$ is quasi-dense in $W$.

Lemma 2.5 ([14, Lemma $2.3(3)])$. Let $(W, S)$ be a Coxeter system and $s, t \in S$ such that $o(s t)=\infty$. Then $W^{\{s\}} t \subset W^{\{t\}}$.

Next, we provide some lemmas for right-angled Coxeter groups. We note that right-angled Coxeter groups are rigid; that is, a right-angled Coxeter group determines its Coxeter system uniquely up to isomorphism ([21]).

By a consequence of Tits' solution to the word problem ([23], [5, p.50]), we can obtain the following lemma (cf. [12, Lemma 5]).

Lemma 2.6. Let $(W, S)$ be a right-angled Coxeter system, let $w \in W$, let $w=$ $s_{1} \cdots s_{l}$ be a reduced representation and let $t, t^{\prime} \in S$. If $t w=t\left(s_{1} \cdots s_{l}\right)$ is reduced and $t w t^{\prime}=w$, then $t=t^{\prime}$ and $t s_{i}=s_{i}$ t for any $i \in\{1, \ldots, l\}$.

Using Lemma 2.6, we prove the following lemma.

Lemma 2.7. Let $(W, S)$ be a right-angled Coxeter system, let $U$ be a spherical subset of $S$, let $s_{0} \in S \backslash U$ and let $T=\left\{t \in U \mid o\left(s_{0} t\right)=2\right\}$. Then $W^{U} s_{0} \subset W^{T \cup\left\{s_{0}\right\}}$.

Proof. Let $w \in W^{U}$. To prove that $w s_{0} \in W^{T \cup\left\{s_{0}\right\}}$, we show that $S\left(w s_{0}\right)=$ $T \cup\left\{s_{0}\right\}$. We note that $\ell\left(w s_{0}\right)=\ell(w)+1$ since $s_{0} \notin U=S(w)$. Hence $s_{0} \in S\left(w s_{0}\right)$. Also for each $t \in T$, by the definition of $T, \ell\left(w s_{0} t\right)=\ell\left(w t s_{0}\right)<\ell\left(w s_{0}\right)$, and $t \in S\left(w s_{0}\right)$. Thus $T \cup\left\{s_{0}\right\} \subset S\left(w s_{0}\right)$. Next we show that $S\left(w s_{0}\right) \subset T \cup\left\{s_{0}\right\}$. Let $t \in S\left(w s_{0}\right)$. Then $\ell\left(w s_{0} t\right)<\ell\left(w s_{0}\right)$. If $w=a_{1} \ldots a_{l}$ is a reduced representation, then by Lemma 2.3 (1),

$$
w s_{0} t=\left(a_{1} \ldots a_{l}\right) s_{0} t=\left(a_{1} \ldots \hat{a_{i}} \ldots a_{l}\right) s_{0}
$$


for some $i \in\{1, \ldots, l\}$, or $t=s_{0}$. By Lemma 2.6. we obtain that $s_{0} t=t s_{0}$. This implies that if $t \neq s_{0}$, then $\ell(w t)<\ell(w)$, i.e., $t \in S(w)=U$. Since $t \in U$ and $s_{0} t=t s_{0}, t \in T$. Hence $S\left(w s_{0}\right) \subset T \cup\left\{s_{0}\right\}$. Thus $S\left(w s_{0}\right)=T \cup\left\{s_{0}\right\}$ and $w s_{0} \in W^{T \cup\left\{s_{0}\right\}}$. We obtain that $W^{U} s_{0} \subset W^{T \cup\left\{s_{0}\right\}}$.

It is well-known that a Coxeter system $(W, S)$ is irreducible if and only if the underlying graph of its Coxeter graph is connected ([1, [5, p.23], [18, p.30]). If the Coxeter system $(W, S)$ is right-angled, then the underlying graph of its Coxeter graph is the graph $\Gamma_{\infty}(W, S)$, where $\Gamma_{\infty}(W, S)$ is defined as follows: the vertex set of $\Gamma_{\infty}(W, S)$ is $S$ and for $s, t \in S,\{s, t\}$ spans an edge in $\Gamma_{\infty}(W, S)$ if and only if $m(s, t)=\infty$. Hence we obtain the following lemma.

Lemma 2.8 (cf. 1], 5], 18]). For a right-angled Coxeter system $(W, S)$, the following statements are equivalent:

(1) $(W, S)$ is irreducible.

(2) $\Gamma_{\infty}(W, S)$ is connected.

(3) For each $a, b \in S$ with $a \neq b$, there exists a sequence $\left\{a=s_{1}, s_{2}, \ldots, s_{n}=\right.$ $b\} \subset S$ such that $o\left(s_{i} s_{i+1}\right)=\infty$ for any $i \in\{1, \ldots, n-1\}$.

\section{Minimality of the Boundary of A Coxeter system}

In this section, we show an extension of a result in [14] on minimality of the boundary of a Coxeter system.

Theorem 3.1. Let $(W, S)$ be a Coxeter system. Suppose that $W^{\left\{s_{0}\right\}}$ is quasi-dense in $W$ and $o\left(s_{0} t_{0}\right)=\infty$ for some $s_{0}, t_{0} \in S$. Then

(1) $\partial \Sigma(W, S)$ is minimal, and

(2) $\left\{w^{\infty} \mid w \in W, o(w)=\infty\right\}$ is dense in $\partial \Sigma(W, S)$.

Proof. Suppose that $W^{\left\{s_{0}\right\}}$ is quasi-dense in $W$ and $o\left(s_{0} t_{0}\right)=\infty$ for some $s_{0}, t_{0} \in S$. Then we show that $W \gamma$ is dense in $\partial \Sigma(W, S)$ for any $\gamma \in \partial \Sigma(W, S)$.

Let $\gamma \in \partial \Sigma(W, S)$ and let $\left\{v_{i}\right\} \subset W$ be a sequence which converges to $\gamma$ in $\Sigma(W, S) \cup \partial \Sigma(W, S)$. Since $W^{\left\{s_{0}\right\}}$ is quasi-dense in $W$, there exists a number $N>0$ such that for each $v \in W, d_{\ell}(v, x) \leq N$ for some $x \in W^{\left\{s_{0}\right\}}$. Hence for each $v \in W$, there exists $u \in W$ such that $\ell(u) \leq N$ and $v u \in W^{\left\{s_{0}\right\}}$. For each $i$, there exists $u_{i} \in W$ such that $\ell\left(u_{i}\right) \leq N$ and $\left(v_{i}\right)^{-1} u_{i} \in W^{\left\{s_{0}\right\}}$. We note that the set $\{u \in W \mid \ell(u) \leq N\}$ is finite because $S$ is finite. Hence $\left\{u_{i} \mid i \in \mathbb{N}\right\}$ is finite, and there exist $u \in W$ and a sequence $\left\{i_{j} \mid j \in \mathbb{N}\right\} \subset \mathbb{N}$ such that $u_{i_{j}}=u$ for any $j \in \mathbb{N}$. Then for each $j \in \mathbb{N},\left(v_{i_{j}}\right)^{-1} u_{i_{j}}=\left(v_{i_{j}}\right)^{-1} u \in W^{\left\{s_{0}\right\}}$ and $\left(v_{i_{j}}\right)^{-1} u t_{0} \in W^{\left\{t_{0}\right\}}$ by Lemma 2.5, since $o\left(s_{0} t_{0}\right)=\infty$. Hence $t_{0} u^{-1} v_{i_{j}} \in\left(W^{\left\{t_{0}\right\}}\right)^{-1}$. The sequence $\left\{t_{0} u^{-1} v_{i_{j}} \mid j \in \mathbb{N}\right\}$ converges to $t_{0} u^{-1} \gamma$, since $\left\{v_{i_{j}} \mid j \in \mathbb{N}\right\}$ converges to $\gamma$. Here we recall the proof of [14, Theorem 4.1]. If we put $x_{j}=t_{0} u^{-1} v_{i_{j}}$ and $\alpha=t_{0} u^{-1} \gamma$, then the sequence $\left\{x_{j}\right\} \subset\left(W^{\left\{t_{0}\right\}}\right)^{-1}$ converges to $\alpha$. By the proof of [14, Theorem 4.1], we obtain that $W \alpha$ is dense in $\partial \Sigma(W, S)$; that is, $W t_{0} u^{-1} \gamma$ is dense in $\partial \Sigma(W, S)$. Hence $W \gamma$ is dense in $\partial \Sigma(W, S)$, since $W t_{0} u^{-1}=W$. Thus every orbit $W \gamma$ is dense in $\partial \Sigma(W, S)$ and $\partial \Sigma(W, S)$ is minimal.

The minimality of $\partial \Sigma(W, S)$ implies that the set $\left\{w^{\infty} \mid w \in W, o(w)=\infty\right\}$ is dense in $\partial \Sigma(W, S)$ (see Proposition 6.2). 
Here we have a question whether conversely if $\partial \Sigma(W, S)$ is minimal, then $W^{\left\{s_{0}\right\}}$ is quasi-dense in $W$ and $o\left(s_{0} t_{0}\right)=\infty$ for some $s_{0}, t_{0} \in S$. The answer to this question is no in general.

For example, let $S=\left\{s_{1}, s_{2}, s_{3}\right\}$ and let

$$
W=\left\langle S \mid s_{1}^{2}=s_{2}^{2}=s_{3}^{2}=\left(s_{1} s_{2}\right)^{4}=\left(s_{2} s_{3}\right)^{4}=\left(s_{3} s_{1}\right)^{4}=1\right\rangle .
$$

Then $W$ is a negatively curved group and the boundary $\partial \Sigma(W, S)$ is minimal. On the other hand, there do not exist $s_{0}, t_{0} \in S$ such that $o\left(s_{0} t_{0}\right)=\infty$.

In Section 5, we will show that in the case $(W, S)$ is right-angled, the answer to this question is yes.

\section{KeY LEMMA}

In this section, we prove the following lemma, which plays a key role in the proof of the main theorem.

Lemma 4.1. Let $(W, S)$ be a right-angled Coxeter system such that $W$ is infinite. If $W$ is irreducible, then $W^{\left\{s_{0}\right\}}$ is quasi-dense in $W$ for some $s_{0} \in S$.

Proof. We suppose that $W^{\{s\}}$ is not quasi-dense in $W$ for any $s \in S$. Then we show that $W$ is not irreducible.

Let $s_{0} \in S$, let $T_{1}=\left\{t \in S \mid o\left(s_{0} t\right)=2\right\}$ and let $S_{1}=S \backslash T_{1}$. If $T_{1}=\emptyset$, then $o\left(s_{0} s\right)=\infty$ for each $s \in S \backslash\left\{s_{0}\right\}$; hence $W^{\left\{s_{0}\right\}}$ is quasi-dense in $W$, which contradicts the assumption. Thus $T_{1} \neq \emptyset$. If $S_{1}=\left\{s_{0}\right\}$, then $W=W_{\left\{s_{0}\right\}} \times W_{T_{1}}$; i.e., $W$ is not irreducible. We suppose that $S_{1} \neq\left\{s_{0}\right\}$.

Let $s_{1} \in S_{1} \backslash\left\{s_{0}\right\}$, let $T_{2}=\left\{t \in T_{1} \mid o\left(s_{1} t\right)=2\right\}$ and let $S_{2}=S \backslash T_{2}=$ $S_{1} \cup\left(T_{1} \backslash T_{2}\right)$. We note that $o\left(s_{i} t\right)=2$ for each $i \in\{0,1\}$ and $t \in T_{2}$, i.e., $W_{\left\{s_{0}, s_{1}\right\} \cup T_{2}}=W_{\left\{s_{0}, s_{1}\right\}} \times W_{T_{2}}$. Since $s_{1} \in S_{1} \backslash\left\{s_{0}\right\}$, we obtain that $o\left(s_{0} s_{1}\right)=\infty$ and $W_{\left\{s_{0}, s_{1}\right\}}$ is irreducible.

Now we show that $T_{2} \neq \emptyset$. Suppose that $T_{2}=\emptyset$. This means that $o\left(s_{1} t\right)=\infty$ for any $t \in T_{1}$. Let $U$ be a maximal spherical subset of $S$ such that $s_{0} \in U$. Then $o(u v)=2$ for each $u, v \in U$ with $u \neq v$, because $(W, S)$ is right-angled and $W_{U}$ is finite. Hence $o\left(s_{0} u\right)=2$ for any $u \in U$, since $s_{0} \in U$. This means that $U \subset T_{1} \cup\left\{s_{0}\right\}$. Hence $o\left(s_{1} u\right)=\infty$ for any $u \in U$, because $o\left(s_{1} t\right)=\infty$ for any $t \in T_{1}$ and $o\left(s_{0} s_{1}\right)=\infty$. Thus $W^{U} s_{1} \subset W^{\left\{s_{1}\right\}}$ by Lemma 2.7. Here by Lemma 2.4. $W^{U}$ is quasi-dense in $W$, since $U$ is a maximal spherical subset of $S$. Hence $W^{\left\{s_{1}\right\}}$ is quasi-dense in $W$. This contradicts the assumption. Thus we obtain that $T_{2} \neq \emptyset$.

If $S_{2}=\left\{s_{0}, s_{1}\right\}$, then $W=W_{\left\{s_{0}, s_{1}\right\}} \times W_{T_{2}}$ and $W$ is not irreducible. We suppose that $S_{2} \neq\left\{s_{0}, s_{1}\right\}$. Let $s_{2} \in S_{2} \backslash\left\{s_{0}, s_{1}\right\}$, let $T_{3}=\left\{t \in T_{2} \mid o\left(s_{2} t\right)=2\right\}$ and let $S_{3}=S \backslash T_{3}=S_{2} \cup\left(T_{2} \backslash T_{3}\right)$.

By induction, we define $s_{k}, T_{k+1}, S_{k+1}$ as follows: Let

$$
\begin{aligned}
& s_{k} \in S_{k} \backslash\left\{s_{0}, \ldots, s_{k-1}\right\}, \\
& T_{k+1}=\left\{t \in T_{k} \mid o\left(s_{k} t\right)=2\right\} \text { and } \\
& S_{k+1}=S \backslash T_{k+1} .
\end{aligned}
$$

Then $W_{\left\{s_{0}, s_{1}, \ldots, s_{k}\right\} \cup T_{k+1}}=W_{\left\{s_{0}, s_{1}, \ldots, s_{k}\right\}} \times W_{T_{k+1}}$. If $S_{k+1} \backslash\left\{s_{0}, s_{1}, \ldots, s_{k}\right\}=\emptyset$, then $W=W_{S_{k+1}} \times W_{T_{k+1}}$, i.e., $W$ is not irreducible. Here we note that $T_{k+1} \subset$ $T_{k} \subset \cdots \subset T_{2} \subset T_{1}$. If $T_{k} \neq \emptyset$ for each $k$, then by the finiteness of $S$, there exists a number $n$ such that $W=W_{S_{n}} \times W_{T_{n}}$; hence $W$ is not irreducible.

We prove the following statements by induction on $k$. 
(i $\left.\mathrm{i}_{\mathrm{k}}\right) T_{k} \neq \emptyset$.

(ii $\mathrm{i}_{\mathrm{k}} W_{\left\{s_{0}, \ldots, s_{k-1}\right\}}$ is irreducible.

(iii $i_{\mathrm{k}}$ ) There exists a spherical subset $U_{k} \subset T_{k}$ such that $W^{U_{k} \cup\left\{s_{i}\right\}}$ is quasi-dense in $W$ for each $i \in\{0, \ldots, k-1\}$.

We first consider the case $k=2$. The statement $\left(\mathrm{i}_{2}\right) T_{2} \neq \emptyset$ was proved in the above. Also (ii 2 ) holds, since $W_{\left\{s_{0}, s_{1}\right\}}=W_{\left\{s_{0}\right\}} * W_{\left\{s_{1}\right\}}$ is irreducible. We show that the statement ( $\mathrm{iii}_{2}$ ) holds. Let $U$ be a maximal spherical subset of $S$ such that $s_{0} \in U$. Then $W^{U}$ is quasi-dense in $W$ by Lemma 2.4. Let $U_{2}=U \cap T_{2}$. We note that $U_{2}=\left\{t \in U \mid o\left(s_{1} t\right)=2\right\}$. By Lemma 2.7 $W^{U} s_{1} \subset W^{U_{2} \cup\left\{s_{1}\right\}}$. Hence $W^{U_{2} \cup\left\{s_{1}\right\}}$ is quasi-dense in $W$. (This implies that $U_{2} \neq \emptyset$ by the assumption.) Also $W^{U_{2} \cup\left\{s_{0}\right\}}$ is quasi-dense in $W$, since $W^{U_{2} \cup\left\{s_{1}\right\}} s_{0} \subset W^{U_{2} \cup\left\{s_{0}\right\}}$ by Lemma 2.7. Thus (iii 2 ) holds.

We suppose that $\left(\mathrm{i}_{\mathrm{k}}\right),\left(\mathrm{ii}_{\mathrm{k}}\right)$ and $\left(\mathrm{iii}_{\mathrm{k}}\right)$ hold for some $k \geq 2$. Then we prove that $\left(\mathrm{i}_{\mathrm{k}+1}\right),\left(\mathrm{ii}_{\mathrm{k}+1}\right)$ and $\left(\mathrm{iii}_{\mathrm{k}+1}\right)$ hold.

$\left(\mathrm{i}_{\mathrm{k}+1}\right)$ : We show that $T_{k+1} \neq \emptyset$. Suppose that $T_{k+1}=\emptyset$. If $o\left(s_{k} s_{i}\right)=2$ for any $i \in\{0,1, \ldots, k-1\}$, then $s_{k} \in T_{k}$, which contradicts the definition of $s_{k}$. Hence $o\left(s_{k} s_{i_{0}}\right)=\infty$ for some $i_{0} \in\{0,1, \ldots, k-1\}$. Since $T_{k+1}=\emptyset, o\left(s_{k} t\right)=\infty$ for any $t \in T_{k}$. Here $U_{k} \subset T_{k}$ and $o\left(s_{k} t\right)=\infty$ for any $t \in U_{k}$. Hence $W^{U_{k} \cup\left\{s_{i_{0}}\right\}} s_{k} \subset W^{\left\{s_{k}\right\}}$ by Lemma 2.7. By (iii $\mathrm{k}$ ), $W^{U} \cup\left\{s_{i_{0}}\right\}$ is quasi-dense in $W$. Thus $W^{\left\{s_{k}\right\}}$ is also quasi-dense in $W$, which contradicts the assumption. Hence $T_{k+1} \neq \emptyset$.

$\left(\mathrm{ii}_{\mathrm{k}+1}\right)$ : We show that $W_{\left\{s_{0}, \ldots, s_{k-1}, s_{k}\right\}}$ is irreducible. Now $o\left(s_{k} s_{i_{0}}\right)=\infty$ for some $i_{0} \in\{0,1, \ldots, k-1\}$ by the above argument. Also $W_{\left\{s_{0}, \ldots, s_{k-1}\right\}}$ is irreducible by the hypothesis $\left(\mathrm{ii}_{\mathrm{k}}\right)$. Hence $W_{\left\{s_{0}, \ldots, s_{k-1}, s_{k}\right\}}$ is irreducible.

$\left(\mathrm{iii}_{\mathrm{k}+1}\right)$ : By $\left(\mathrm{iii}_{\mathrm{k}}\right)$, there exists a spherical subset $U_{k} \subset T_{k}$ such that $W^{U} \cup\left\{s_{i}\right\}$ is quasi-dense in $W$ for each $i \in\{0, \ldots, k-1\}$. We define $U_{k+1}=U_{k} \cap T_{k+1}$, i.e., $U_{k+1}=\left\{t \in U_{k} \mid o\left(s_{k} t\right)=2\right\}$. Here $o\left(s_{k} s_{i_{0}}\right)=\infty$ for some $i_{0} \in\{0,1, \ldots$, $k-1\}$ by the above argument. Then $W^{U_{k} \cup\left\{s_{i_{0}}\right\}_{k}} \subset W^{U_{k+1} \cup\left\{s_{k}\right\}}$ by Lemma 2.7. Hence $W^{U_{k+1} \cup\left\{s_{k}\right\}}$ is quasi-dense in $W$, since $W^{U} \cup\left\{s_{i_{0}}\right\}$ is so. Finally we show that $W^{U_{k+1} \cup\left\{s_{i}\right\}}$ is quasi-dense in $W$ for each $i \in\{0, \ldots, k-1, k\}$. We note that $W_{\left\{s_{0}, \ldots, s_{k-1}, s_{k}\right\}}$ is irreducible by $\left(\mathrm{ii}_{\mathrm{k}+1}\right)$. Hence for each $j_{0} \in\{0, \ldots, k-1\}$, there exists a sequence $\left\{s_{k}=a_{0}, a_{1}, \ldots, a_{m}=s_{j_{0}}\right\} \subset\left\{s_{i} \mid i=0,1, \ldots, k\right\}$ such that $o\left(a_{i} a_{i+1}\right)=\infty$ by Lemma 2.8. Then by Lemma 2.7.

$$
\begin{aligned}
W^{U_{k+1} \cup\left\{s_{k}\right\}} a_{1} a_{2} \cdots a_{m} & \subset W^{U_{k+1} \cup\left\{a_{1}\right\}} a_{2} \cdots a_{m} \\
& \subset \cdots \subset W^{U_{k+1} \cup\left\{a_{m}\right\}}=W^{U_{k+1} \cup\left\{s_{j_{0}}\right\},}
\end{aligned}
$$

because $o\left(s_{i} u\right)=2$ for any $i \in\{0,1, \ldots, k-1, k\}$ and $u \in U_{k+1}$. Thus $W^{U_{k+1} \cup\left\{s_{j_{0}}\right\}}$ is quasi-dense in $W$. Hence ( iii $_{\mathrm{k}+1}$ ) holds.

Thus by the induction on $k$, we can define $s_{k-1}, T_{k}, S_{k}$ which satisfy $\left(\mathrm{i}_{\mathrm{k}}\right),\left(\mathrm{ii}_{\mathrm{k}}\right)$ and (iii ${ }_{\mathrm{k}}$ ). Since $S$ is finite, there exists a number $n$ such that $S_{n}=\left\{s_{0}, s_{1}, \ldots, s_{n-1}\right\}$ and $W=W_{S_{n}} \times W_{T_{n}}$, where $T_{n} \neq \emptyset$. Therefore $W$ is not irreducible.

\section{Dense subsets of the Boundary of A Right-Angled Coxeter group}

We obtain the following main theorem from Theorem 3.1 and Lemma 4.1

Theorem 5.1. Let $(W, S)$ be a right-angled Coxeter system such that $W$ is infinite. Then the following statements are equivalent:

(1) $\partial \Sigma(W, S)$ is minimal. 
(2) $W_{\tilde{S}}$ is irreducible.

(3) $W^{\left\{s_{0}\right\}}$ is quasi-dense in $W$ and $o\left(s_{0} t_{0}\right)=\infty$ for some $s_{0}, t_{0} \in S$.

(4) There does not exist a finite-index subgroup of $W$ which splits as a product $W_{1} \times W_{2}$ where each $W_{i}$ is infinite.

Proof. (3) $\Rightarrow(1)$ : If the statement (3) holds, then $\partial \Sigma(W, S)$ is minimal by Theorem 3.1

$(1) \Rightarrow(2)$ : Suppose that $W_{\tilde{S}}$ is not irreducible. Let $W_{\tilde{S}}=W_{S_{1}} \times W_{S_{2}}$, where $W_{S_{1}}$ and $W_{S_{2}}$ are infinite. Then $\partial \Sigma(W, S)=\partial \Sigma\left(W_{\tilde{S}}, \tilde{S}\right)$ and $\Sigma\left(W_{\tilde{S}}, \tilde{S}\right)=\Sigma\left(W_{S_{1}}, S_{1}\right) \times$ $\Sigma\left(W_{S_{2}}, S_{2}\right)$. Here by [11, Theorem 4.3], $\partial \Sigma\left(W_{S_{1}}, S_{1}\right)$ is $W$-invariant, that is, $W \partial \Sigma\left(W_{S_{1}}, S_{1}\right)=\partial \Sigma\left(W_{S_{1}}, S_{1}\right)$. Thus for $\alpha \in \partial \Sigma\left(W_{S_{1}}, S_{1}\right), \overline{W \alpha} \subset \partial \Sigma\left(W_{S_{1}}, S_{1}\right)$. Hence $\partial \Sigma(W, S)$ is not minimal. In Section 6 , we will give a more general proof (Theorem 6.4).

$(2) \Rightarrow(3)$ : Suppose that $W_{\tilde{S}}$ is irreducible. By Lemma 4.1, $\left(W_{\tilde{S}}\right)^{\left\{s_{0}\right\}}=W^{\left\{s_{0}\right\}} \cap$ $W_{\tilde{S}}$ is quasi-dense in $W_{\tilde{S}}$ for some $s_{0} \in \tilde{S}$. Here $W=W_{\tilde{S}} \times W_{S \backslash \tilde{S}}$ and $W_{S \backslash \tilde{S}}$ is finite (see [11]). Hence $W^{\left\{s_{0}\right\}}$ is quasi-dense in $W$. Since $W_{\tilde{S}}$ is irreducible, $o\left(s_{0} t_{0}\right)=\infty$ for some $t_{0} \in \tilde{S}$ by Lemma 2.8. Thus the statement (3) holds.

$(4) \Rightarrow(2)$ : If $W_{\tilde{S}}$ is not irreducible, then $W_{\tilde{S}}$ splits as a product $W_{\tilde{S}}=W_{A_{1}} \times W_{A_{2}}$ for some $A_{i} \subset \tilde{S}$, where each $W_{A_{i}}$ is infinite. Here $W_{\tilde{S}}$ is a finite-index subgroup of $W$.

$(1) \Rightarrow(4)$ : We obtain this implication from Theorem 6.4.

The following question appears in [15].

Question 5.2. Let $(W, S)$ be a Coxeter system. Is it the case that if $(W, S)$ is an irreducible Coxeter system, then $W \partial \Sigma\left(W_{T}, T\right)$ is dense in $\partial \Sigma(W, S)$ for any subset $T$ of $S$ such that $W_{T}$ is infinite?

Theorem 5.1 implies that the answer to Question 5.2 is yes for right-angled Coxeter groups. Moreover, as an application of Theorem [5.1] we obtain the following corollary.

Corollary 5.3. Let $(W, S)$ be a right-angled Coxeter system and let $T \subset S$. Then the following statements are equivalent:

(1) $W \partial \Sigma\left(W_{T}, T\right)$ is dense in $\partial \Sigma(W, S)$.

(2) If $W=W_{S_{1}} \times \cdots \times W_{S_{n}}$ is the irreducible decomposition of $W$, then $W_{S_{i} \cap T}$ is infinite for each $i \in\{1, \ldots, n\}$ such that $W_{S_{i}}$ is infinite.

Proof. (1) $\Rightarrow$ (2): Let $W=W_{S_{1}} \times \cdots \times W_{S_{n}}$ be the irreducible decomposition of $W$. We suppose that there exists $i_{0} \in\{1, \ldots, n\}$ such that $W_{S_{i_{0}}}$ is infinite and $W_{S_{i_{0}} \cap T}$ is finite. Let $A_{1}=S \backslash S_{i_{0}}$ and $A_{2}=S_{i_{0}}$. Then $W=W_{A_{1}} \times W_{A_{2}}, W_{A_{2}}$ is infinite and $W_{A_{2} \cap T}$ is finite. We note that $\partial \Sigma\left(W_{A_{1}}, A_{1}\right)$ is $W$-invariant by [11, Theorem 4.3]. Since $W_{T}=W_{A_{1} \cap T} \times W_{A_{2} \cap T}$ and $W_{A_{2} \cap T}$ is finite, $\partial \Sigma\left(W_{T}, T\right) \subset \partial \Sigma\left(W_{A_{1}}, A_{1}\right)$. Thus

Since $W_{A_{2}}$ is infinite and

$$
W \partial \Sigma\left(W_{T}, T\right) \subset W \partial \Sigma\left(W_{A_{1}}, A_{1}\right)=\partial \Sigma\left(W_{A_{1}}, A_{1}\right) .
$$

$$
\partial \Sigma(W, S)=\partial \Sigma\left(W_{A_{1}}, A_{1}\right) * \partial \Sigma\left(W_{A_{2}}, A_{2}\right)
$$

$W \partial \Sigma\left(W_{T}, T\right)$ is not dense in $\partial \Sigma(W, S)$.

$(2) \Rightarrow(1)$ : Let $W=W_{S_{1}} \times \cdots \times W_{S_{n}}$ be the irreducible decomposition of $W$. Suppose that (2) holds. Then we prove that (1) holds by induction on $n$. 
We first consider the case $n=1$. Then $W=W_{S_{1}}$ is irreducible. Since $W_{S_{1} \cap T}$ is infinite, $\partial \Sigma\left(W_{T}, T\right) \neq \emptyset$. Hence $W \partial \Sigma\left(W_{T}, T\right)$ is dense in $\partial \Sigma(W, S)$ by Theorem 5.1.

Next we consider the case $n>1$. Let $A_{1}=S_{1} \cup \cdots \cup S_{n-1}$ and $A_{2}=S_{n}$. Then $W=W_{A_{1}} \times W_{A_{2}}$ and $W_{T}=W_{A_{1} \cap T} \times W_{A_{2} \cap T}$. Here

$$
\begin{aligned}
W \partial \Sigma\left(W_{T}, T\right) & =W\left(\partial \Sigma\left(W_{A_{1} \cap T}, A_{1} \cap T\right) * \partial \Sigma\left(W_{A_{2} \cap T}, A_{2} \cap T\right)\right) \\
& \supset W_{A_{1}} \partial \Sigma\left(W_{A_{1} \cap T}, A_{1} \cap T\right) * W_{A_{2}} \partial \Sigma\left(W_{A_{2} \cap T}, A_{2} \cap T\right) .
\end{aligned}
$$

By the inductive hypothesis, $W_{A_{i}} \partial \Sigma\left(W_{A_{i} \cap T}, A_{i} \cap T\right)$ is dense in $\partial \Sigma\left(W_{A_{i}}, A_{i}\right)$ for each $i=1,2$. Since

$$
\partial \Sigma(W, S)=\partial \Sigma\left(W_{A_{1}}, A_{1}\right) * \partial \Sigma\left(W_{A_{2}}, A_{2}\right)
$$

we obtain that $W \partial \Sigma\left(W_{T}, T\right)$ is dense in $\partial \Sigma(W, S)$.

Also we obtain the following corollary from Theorem 5.1. We give a proof in Section 6 .

Corollary 5.4. For a right-angled Coxeter system $(W, S)$, the set $\left\{w^{\infty} \mid w \in\right.$ $W, o(w)=\infty\}$ is dense in the boundary $\partial \Sigma(W, S)$.

\section{Remarks on Dense subsets of boundaries of CAT(0) Groups}

In this section, we investigate dense subsets of boundaries of CAT(0) groups. The definitions and basic properties of $\mathrm{CAT}(0)$ spaces and their boundaries can be found in [4. A group $\Gamma$ is called a $C A T(0)$ group if $\Gamma$ acts geometrically (i.e., properly and cocompactly by isometries) on some CAT(0) space. For example, a Coxeter group $W$ acts geometrically on the Davis complex $\Sigma(W, S)$, which is a $\mathrm{CAT}(0)$ space, and every Coxeter group is a $\mathrm{CAT}(0)$ group.

We pose the following open problem.

Question 6.1. Suppose that a group $\Gamma$ acts geometrically on a CAT(0) space $X$. Is it the case that the set $\left\{\gamma^{\infty} \mid \gamma \in \Gamma, o(\gamma)=\infty\right\}$ is dense in the boundary $\partial X$ ?

Here we note that $\gamma^{\infty}$ is the point of the boundary $\partial X$ to which the sequence $\left\{\gamma^{i} x_{0} \mid i \in \mathbb{N}\right\} \subset X$ converges in $X \cup \partial X$, where $x_{0} \in X$ and $\gamma^{\infty}$ does not depend on the point $x_{0}$.

We introduce some relations between this question and the minimality of boundaries of $\mathrm{CAT}(0)$ groups.

We first show the following proposition.

Proposition 6.2. Suppose that a group $\Gamma$ acts geometrically on a CAT(0) space $X$. If there exists $\delta \in \Gamma$ such that $o(\delta)=\infty$ and $\Gamma \delta^{\infty}$ is dense in the boundary $\partial X$, then the set $\left\{\gamma^{\infty} \mid \gamma \in \Gamma, o(\gamma)=\infty\right\}$ is dense in $\partial X$. Hence, if the boundary $\partial X$ is minimal, then the set $\left\{\gamma^{\infty} \mid \gamma \in \Gamma, o(\gamma)=\infty\right\}$ is dense in $\partial X$.

Proof. Suppose that $\delta \in \Gamma$ such that $o(\delta)=\infty$ and $\Gamma \delta^{\infty}$ is dense in $\partial X$. Let $\alpha \in \partial X$. Since $\Gamma \delta^{\infty}$ is dense in $\partial X$, there exists a sequence $\left\{\gamma_{i}\right\} \subset \Gamma$ such that $\left\{\gamma_{i} \delta^{\infty}\right\}$ converges to $\alpha$ in $\partial X$. Here for $x_{0} \in X$ and each $i$, the sequence $\left\{\left(\gamma_{i} \delta \gamma_{i}^{-1}\right)^{j} x_{0}\right\}_{j}$ converges to $\gamma_{i} \delta^{\infty}$ in $X \cup \partial X$. Hence $\left(\gamma_{i} \delta \gamma_{i}^{-1}\right)^{\infty}=\gamma_{i} \delta^{\infty}$ and $\left\{\left(\gamma_{i} \delta \gamma_{i}^{-1}\right)^{\infty}\right\}_{i}$ converges to $\alpha$ in $\partial X$. Thus $\left\{\gamma^{\infty} \mid \gamma \in \Gamma, o(\gamma)=\infty\right\}$ is dense in $\partial X$.

Now we suppose that the boundary $\partial X$ is minimal. It is known that every $\operatorname{CAT}(0)$ group has an element of infinite order (22, Theorem 11]). Let $\delta \in \Gamma$ with $o(\delta)=\infty$. Then $\Gamma \delta^{\infty}$ is dense in $\partial X$ because $\partial X$ is minimal. Hence, by the above argument, the set $\left\{\gamma^{\infty} \mid \gamma \in \Gamma, o(\gamma)=\infty\right\}$ is dense in the boundary $\partial X$. 
We obtain the following proposition from some splitting theorems for $\operatorname{CAT}(0)$ spaces.

Proposition 6.3. Suppose that a group $\Gamma=\Gamma_{1} \times \Gamma_{2}$ acts geometrically on a CAT(0) space $X$, where $\Gamma_{1}$ and $\Gamma_{2}$ are infinite. Then $X$ contains a quasi-dense subspace $X^{\prime}=X_{1} \times X_{2}$ and there exists a product subgroup $\Gamma_{1}^{\prime} \times \Gamma_{2}^{\prime}$ of finite index in $\Gamma$ such that $X_{1}$ is the convex hull $C\left(\Gamma_{1}^{\prime} x_{0}\right)$ for some $x_{0} \in X$ and $\Gamma_{2}^{\prime}$ acts geometrically on $X_{2}$ by projection.

Proof. By [13, Lemma 2.1], there exist subgroups $G_{1} \times A_{1}$ and $G_{2} \times A_{2}$ of finite index in $\Gamma_{1}$ and $\Gamma_{2}$ respectively such that $G_{1}$ and $G_{2}$ have finite center and $A_{i}$ is isomorphic to $\mathbb{Z}^{n_{i}}$ for some $n_{i}(i=1,2)$.

In the case that $A_{i}$ is not trivial for some $i \in\{1,2\}$, we put $\Gamma_{1}^{\prime}=A_{i}$ and $\Gamma_{2}^{\prime}=G_{1} \times G_{2} \times A_{3-i}$. Then by [3, Proposition 1.1] and [4, Theorem II.7.1], the proposition holds.

In the case that $A_{1}$ and $A_{2}$ are trivial, we put $\Gamma_{1}^{\prime}=G_{1}$ and $\Gamma_{2}^{\prime}=G_{2}$. Here $G_{1}$ and $G_{2}$ have finite center. By [17, Theorem 2] and [19, Corollary 10], the proposition holds. Here concerning the condition in [19, Corollary 10], we note that if the $\operatorname{CAT}(0)$ group $\Gamma$ has finite center, then there does not exist a $\Gamma$-fixed point in the boundary $\partial X$ (cf. [17, Lemma 3.2]).

Concerning the nonminimality of boundaries of CAT(0) groups, using Proposition 6.3, we show the following theorem.

Theorem 6.4. Suppose that a group $\Gamma$ acts geometrically on a $C A T(0)$ space $X$. If $\Gamma$ contains a finite-index subgroup $\Gamma_{1} \times \Gamma_{2}$ where $\Gamma_{1}$ and $\Gamma_{2}$ are infinite, then the boundary $\partial X$ is not minimal.

Proof. Let $\Gamma_{1} \times \Gamma_{2}$ be a finite-index subgroup of $\Gamma$, where $\Gamma_{1}$ and $\Gamma_{2}$ are infinite. Then $\Gamma_{1} \times \Gamma_{2}$ acts geometrically on $X$. By Proposition 6.3, $X$ contains a quasi-dense subspace $X_{1} \times X_{2}$ and there exists a product subgroup $\Gamma_{1}^{\prime} \times \Gamma_{2}^{\prime}$ of finite index in $\Gamma$ such that $X_{1}$ is the convex hull $C\left(\Gamma_{1}^{\prime} x_{0}\right)$ for some $x_{0} \in X$ and $\Gamma_{2}^{\prime}$ acts geometrically on $X_{2}$ by projection.

To prove that $\partial X$ is not minimal, we show that $\Gamma\left(\partial X_{1}\right)$ is not dense in $\partial X$.

Since $\Gamma_{1}^{\prime} \times \Gamma_{2}^{\prime}$ is a subgroup of finite index in $\Gamma$, there exist a number $n$ and $\left\{\delta_{1}, \ldots, \delta_{n}\right\} \subset \Gamma$ such that $\Gamma=\bigcup_{i=1}^{n} \delta_{i}\left(\Gamma_{1}^{\prime} \times \Gamma_{2}^{\prime}\right)$.

Since $X_{1}=C\left(\Gamma_{1}^{\prime} x_{0}\right)$ is $\Gamma_{1}^{\prime}$-invariant, $\Gamma_{1}^{\prime}\left(\partial X_{1}\right)=\partial X_{1}$. For each $\gamma_{2} \in \Gamma_{2}^{\prime}, \gamma_{2} X_{1}$ and $X_{1}$ are parallel by the proof of the splitting theorems ([3], [4, [17, [1] ); hence $\gamma_{2}\left(\partial X_{1}\right)=\partial X_{1}$, that is, $\Gamma_{2}^{\prime}\left(\partial X_{1}\right)=\partial X_{1}$. Thus $\left(\Gamma_{1}^{\prime} \times \Gamma_{2}^{\prime}\right)\left(\partial X_{1}\right)=\partial X_{1}$.

Hence

$$
\begin{aligned}
\Gamma\left(\partial X_{1}\right) & =\left(\bigcup_{i=1}^{n} \delta_{i}\left(\Gamma_{1}^{\prime} \times \Gamma_{2}^{\prime}\right)\right)\left(\partial X_{1}\right) \\
& =\bigcup_{i=1}^{n}\left(\delta_{i}\left(\Gamma_{1}^{\prime} \times \Gamma_{2}^{\prime}\right)\left(\partial X_{1}\right)\right) \\
& =\bigcup_{i=1}^{n}\left(\delta_{i}\left(\partial X_{1}\right)\right)
\end{aligned}
$$


Here we note that $\Gamma\left(\partial X_{1}\right)=\bigcup_{i=1}^{n}\left(\delta_{i}\left(\partial X_{1}\right)\right)$ is closed. Hence

$$
\begin{aligned}
\operatorname{dim} \overline{\Gamma\left(\partial X_{1}\right)} & =\operatorname{dim} \bigcup_{i=1}^{n}\left(\delta_{i}\left(\partial X_{1}\right)\right)=\operatorname{dim} \partial X_{1} \\
& <\operatorname{dim}\left(\partial X_{1} \times[0,1]\right) \leq \operatorname{dim}\left(\partial X_{1} * \partial X_{2}\right)=\operatorname{dim} \partial X .
\end{aligned}
$$

Here we note that $\operatorname{dim} \partial X_{i}$ is finite, because the boundary of a cocompact proper $\mathrm{CAT}(0)$ space is finite-dimensional ([22, Theorem 12]).

Thus $\Gamma\left(\partial X_{1}\right)$ is not dense in $\partial X$. This implies that $\partial X$ is not minimal.

The referee has pointed out that the converse of Theorem 6.4 (if $\Gamma$ does not contain a finite-index subgroup $\Gamma_{1} \times \Gamma_{2}$ where $\Gamma_{1}$ and $\Gamma_{2}$ are infinite, then the boundary $\partial X$ is minimal) will not be true in general and that a counterexample will be supplied by the theory of lattices in semisimple groups, since an irreducible lattice on a product of two hyperbolic planes does not factor (with infinite factors) (cf. [10]).

On the other hand, Theorem 5.1 implies that the converse of Theorem 6.4 holds for right-angled Coxeter groups and their boundaries.

Let $\mathcal{A}$ be the set of all infinite $\mathrm{CAT}(0)$ groups $\Gamma$ such that for any $\mathrm{CAT}(0)$ space $X$ on which $\Gamma$ acts geometrically, the set $\left\{\gamma^{\infty} \mid \gamma \in \Gamma, o(\gamma)=\infty\right\}$ is dense in $\partial X$.

Now we show the following proposition.

Proposition 6.5. Suppose that $\Gamma_{1}, \ldots, \Gamma_{n} \in \mathcal{A}$ and that each $\Gamma_{i}$ does not contain a finite-index subgroup $\Gamma_{i 1} \times \Gamma_{i 2}$ such that $\Gamma_{i 1}$ and $\Gamma_{i 2}$ are infinite. Then $\Gamma_{1} \times \cdots \times \Gamma_{n} \in$ $\mathcal{A}$.

Proof. We note that each $\Gamma_{i}$ is either isomorphic to $\mathbb{Z}$ or has finite center by [13, Lemma 2.1]. Hence we can suppose that for some number $k, \Gamma_{i}$ is isomorphic to $\mathbb{Z}$ for each $i \leq k$ and $\Gamma_{i}$ has finite center for each $i>k$.

We prove that $\Gamma \in \mathcal{A}$ by induction on $n$.

In the case $n=1$, it is obvious.

We consider the case $n=2$. Suppose that $\Gamma=\Gamma_{1} \times \Gamma_{2}$ acts geometrically on a CAT(0) space $X$. By Proposition 6.3, $X$ contains a quasi-dense subspace $X_{1} \times X_{2}$ such that $X_{1}=C\left(\Gamma_{1} x_{0}\right)$ for some $x_{0} \in X$ and $\Gamma_{2}$ acts geometrically on $X_{2}$ by projection. Let $\alpha \in \partial X$. Here

$$
\partial X=\partial X_{1} * \partial X_{2}=\left(\partial X_{1} \times \partial X_{2} \times[-\pi, \pi]\right) / \sim .
$$

Hence $\alpha=\left[\alpha_{1}, \alpha_{2}, \theta\right]$ for some $\alpha_{1} \in \partial X_{1}, \alpha_{2} \in \partial X_{2}$ and $\theta \in[-\pi, \pi]$. Now $\left\{\gamma^{\infty} \mid \gamma \in\right.$ $\left.\Gamma_{1}, o(\gamma)=\infty\right\}$ is dense in $\partial X_{1}$ and $\left\{\delta^{\infty} \mid \delta \in \Gamma_{2}, o(\delta)=\infty\right\}$ is dense in $\partial X_{2}$. Hence there exist sequences $\left\{\gamma_{i}\right\} \subset \Gamma_{1}$ and $\left\{\delta_{i}\right\} \subset \Gamma_{2}$ such that $\left\{\gamma_{i}^{\infty}\right\}$ converges to $\alpha_{1}$ and $\left\{\delta_{i}^{\infty}\right\}$ converges to $\alpha_{2}$. Since $\left\langle\gamma_{i}, \delta_{i}\right\rangle$ is isomorphic to $\mathbb{Z} \times \mathbb{Z}$, by the Flat Torus Theorem (4, Theorem II.7.1]), $\left\langle\gamma_{i}, \delta_{i}\right\rangle$ acts geometrically on some convex hull $C\left(\left\langle\gamma_{i}, \delta_{i}\right\rangle x_{i}\right)$ which is isometric to the Euclidean plane. Here $C\left(\left\langle\gamma_{i}, \delta_{i}\right\rangle x_{i}\right) \subset X_{1} \times X_{2}$ and

$$
\left\{\gamma_{i}^{-\infty}, \gamma_{i}^{\infty}, \delta_{i}^{-\infty}, \delta_{i}^{\infty}\right\} \subset \partial\left(C\left(\left\langle\gamma_{i}, \delta_{i}\right\rangle x_{i}\right)\right)
$$

Then there exists a sequence $\left\{a_{i j}\right\} \subset\left\langle\gamma_{i}, \delta_{i}\right\rangle$ such that $\left\{a_{i j}^{\infty}\right\}_{j}$ converges to $\left[\gamma_{i}^{\infty}, \delta_{i}^{\infty}, \theta\right]$. Here the sequence $\left\{\left[\gamma_{i}^{\infty}, \delta_{i}^{\infty}, \theta\right]\right\}_{i}$ converges to $\alpha$. Hence

$$
\alpha \in \overline{\left\{a_{i j}^{\infty} \mid i, j \in \mathbb{N}\right\}} \subset \overline{\left\{\gamma^{\infty} \mid \gamma \in \Gamma, o(\gamma)=\infty\right\}} .
$$

Thus $\left\{\gamma^{\infty} \mid \gamma \in \Gamma, o(\gamma)=\infty\right\}$ is dense in $\partial X$ and $\Gamma=\Gamma_{1} \times \Gamma_{2} \in \mathcal{A}$. 
We consider the case $n>2$. Suppose that $\Gamma=\Gamma_{1} \times \cdots \times \Gamma_{n-1} \times \Gamma_{n}$ acts geometrically on a $\operatorname{CAT}(0)$ space $X$. Let $\tilde{\Gamma}_{1}=\Gamma_{1} \times \cdots \times \Gamma_{n-1}$ and $\tilde{\Gamma}_{2}=\Gamma_{n}$. Here we can suppose that $\tilde{\Gamma}_{2}$ has finite center or that each $\Gamma_{i}$ is isomorphic to $\mathbb{Z}$ for $i=1, \ldots, n$. By the inductive hypothesis and the same argument as the proof in the case $n=2$, we obtain that $\left\{\gamma^{\infty} \mid \gamma \in \Gamma, o(\gamma)=\infty\right\}$ is dense in $\partial X$ and $\Gamma=\Gamma_{1} \times \cdots \times \Gamma_{n-1} \times \Gamma_{n} \in \mathcal{A}$.

Suppose that a group $\Gamma$ acts geometrically on a $\operatorname{CAT}(0)$ space $X$. Then there exists a finite-index subgroup $\Gamma_{1} \times \cdots \times \Gamma_{n}$ of $\Gamma$ such that each $\Gamma_{i}$ is infinite and each $\Gamma_{i}$ does not contain a finite-index subgroup $\Gamma_{i 1} \times \Gamma_{i 2}$ where $\Gamma_{i 1}$ and $\Gamma_{i 2}$ are infinite. Here the decomposition process terminates and $n$ is finite. Indeed each $\Gamma_{i}$ is a CAT(0) group by [17, Theorem 9.1] and there exists $\gamma_{i} \in \Gamma_{i}$ with $o\left(\gamma_{i}\right)=\infty$ by [22, Theorem 11]. Then $\left\langle\gamma_{1}, \ldots, \gamma_{n}\right\rangle \subset \Gamma$ is isomorphic to $\mathbb{Z}^{n}$. Here such an $n$ is finite, because every abelian subgroup of a CAT(0) group is finitely generated (4, Corollary II.7.6]).

Hence Proposition 6.5 implies that Question 6.1 is equivalent to the following question.

Question 6.6. For an infinite $\operatorname{CAT}(0)$ group $\Gamma$ which does not contain a finiteindex product subgroup of two infinite subgroups, does $\Gamma \in \mathcal{A}$ ?

Finally, we prove Corollary 5.4. Concerning Question 6.1, we obtain a positive answer for right-angled Coxeter groups and their boundaries.

Proof of Corollary 5.4. Let $(W, S)$ be a right-angled Coxeter system and let $W=$ $W_{S_{1}} \times \cdots \times W_{S_{n}}$ be the irreducible decomposition of $W$. We may suppose that $W_{S_{i}}$ is infinite for any $i \leq k$ and $W_{S_{i}}$ is finite for any $i>k$ for some number $k$. Then $\tilde{S}=S_{1} \cup \cdots \cup S_{k}$ and

$$
\Sigma(W, S)=\Sigma\left(W_{S_{1}}, S_{1}\right) \times \cdots \times \Sigma\left(W_{S_{k}}, S_{k}\right) \times \Sigma\left(W_{S \backslash \tilde{S}}, S \backslash \tilde{S}\right),
$$

where $\Sigma\left(W_{S \backslash \tilde{S}}, S \backslash \tilde{S}\right)$ is bounded, since $W_{S \backslash \tilde{S}}$ is finite. Hence,

$$
\partial \Sigma(W, S)=\partial \Sigma\left(W_{S_{1}}, S_{1}\right) * \cdots * \partial \Sigma\left(W_{S_{k}}, S_{k}\right) .
$$

Here each Coxeter system $\left(W_{S_{i}}, S_{i}\right)$ is irreducible and right-angled and $\partial \Sigma\left(W_{S_{i}}, S_{i}\right)$ is minimal by Theorem [5.1. Thus for each $i \in\{1, \ldots, k\}$, the set $\left\{w^{\infty} \mid w \in\right.$ $\left.W_{i}, o(w)=\infty\right\}$ is dense in $\partial \Sigma\left(W_{S_{i}}, S_{i}\right)$ by Proposition 6.2. By a similar argument to the proof of Proposition 6.5, we obtain that the set $\left\{w^{\infty} \mid w \in W, o(w)=\infty\right\}$ is dense in the boundary $\partial \Sigma(W, S)$.

\section{ACKNOWLEDGMENT}

The author would like to thank the referee for helpful comments.

\section{REFERENCES}

[1] N. Bourbaki, Groupes et Algebrès de Lie, Chapters IV-VI, Masson, Paris, 1981. MR647314 (83g:17001)

[2] P. L. Bowers and K. Ruane, Fixed points in boundaries of negatively curved groups, Proc. Amer. Math. Soc. 124 (no. 4) (1996), 1311-1313. MR1343682 (96g:20052)

[3] P. L. Bowers and K. Ruane, Boundaries of nonpositively curved groups of the form $G \times \mathbb{Z}^{n}$, Glasgow Math. J. 38 (1996), 177-189. MR1397173 (97d:20039)

[4] M. R. Bridson and A. Haefliger, Metric spaces of non-positive curvature, Springer-Verlag, Berlin, 1999. MR.1744486 (2000k:53038)

[5] K. S. Brown, Buildings, Springer-Verlag, 1980. MR969123 (90e:20001) 
[6] M. W. Davis, Groups generated by reflections and aspherical manifolds not covered by Euclidean space, Ann. of Math. (2) 117 (1983), 293-324. MR690848 (86d:57025)

[7] M. W. Davis, Nonpositive curvature and reflection groups, in Handbook of geometric topology (Edited by R. J. Daverman and R. B. Sher), pp. 373-422, North-Holland, Amsterdam, 2002. MR.1886674(2002m:53061)

[8] M. W. Davis, The cohomology of a Coxeter group with group ring coefficients, Duke Math. J. 91 (no. 2) (1998), 297-314. MR1600586 (99b:20067)

[9] M. Gromov, Hyperbolic groups, in Essays in group theory (Edited by S. M. Gersten), pp. 75263, M.S.R.I. Publ. 8, 1987. MR919829 (89e:20070)

[10] J. A. Hillman, Four-manifolds, geometries and knots, Geometry and Topology Monographs, vol. 5, Geometry and Topology Publications, Coventry, 2002. MR 1943724 (2003m:57047)

[11] T. Hosaka, Parabolic subgroups of finite index in Coxeter groups, J. Pure Appl. Algebra 169 (2002), 215-227. MR.1897344 (2003c:20041)

[12] T. Hosaka, Determination up to isomorphism of right-angled Coxeter systems, Proc. Japan Acad. Ser. A Math. Sci. 79 (2003), 33-35. MR1960740 (2004b:20053)

[13] T. Hosaka, A splitting theorem for CAT(0) spaces with the geodesic extension property, Tsukuba J. Math. 27 (2003), 289-293. MR2025728 (2004j:20089)

[14] T. Hosaka, Dense subsets of the boundary of a Coxeter system, Proc. Amer. Math. Soc. 132 (2004), 3441-3448. MR2073322 (2005g:57004)

[15] T. Hosaka, Addendum to "Dense subsets of the boundary of a Coxeter system", Proc. Amer. Math. Soc. 133 (2005), 3745-3747. MR2163614 (2006c:57002)

[16] T. Hosaka, On dense orbits in the boundary of a Coxeter system, J. Math. Kyoto Univ. 45 (no. 3) (2005), 627-631. MR2206364 (2006k:57004)

[17] T. Hosaka, On splitting theorems for CAT(0) spaces and compact geodesic spaces of nonpositive curvature, preprint, arXiv:math.GR/0405551v1 (2004).

[18] J. E. Humphreys, Reflection groups and Coxeter groups, Cambridge University Press, 1990. MR1066460 (92h:20002)

[19] N. Monod, Superrigidity for irreducible lattices and geometric splitting, J. Amer. Math. Soc. 19 (2006), 781-814. MR2219304 (2007b:22025)

[20] G. Moussong, Hyperbolic Coxeter groups, Ph.D. thesis, Ohio State University, 1988.

[21] D. Radcliffe, Unique presentation of Coxeter groups and related groups, Ph.D. thesis, University of Wisconsin-Milwaukee, 2001.

[22] E. L. Swenson, A cut point theorem for CAT(0) groups, J. Differential Geom. 53 (1999), 327-358. MR.1802725 (2001i:20083)

[23] J. Tits, Le problème des mots dans les groupes de Coxeter, Symposia Mathematica, vol. 1, pp. 175-185, Academic Press, London, 1969. MR0254129 (40:7339)

Department of Mathematics, Faculty of Education, Utsunomiya University, UtsunoMIYA, 321-8505, JAPAN

E-mail address: hosaka@cc.utsunomiya-u.ac.jp 